\title{
Numerical and theoretical treatment based on the compact finite difference and Spectral collocation algorithms of the space fractional-order Fisher's equation
}

Mohamed Adel $^{1}$ and Mohamed Khader ${ }^{2}$

${ }^{1}$ Cairo Univeisty

${ }^{2}$ Benha University

May 5, 2020

\begin{abstract}
This paper present an accurate numerical algorithm to solve the space fractional-order Fisher's equation where the derivative operator is described in the Caputo derivative sense. In the presented discretization process, first we use the compact nite difference (CFD) to occur a semi-discrete in time derivative, and implement the Chebyshev spectral collocation method (CSCM) of the third-kind to discretize the spatial fractional derivative. The presented method converts the studied problem to be a system of algebraic equations which can be easily solved. To study the convergence and stability analysis, some theorems are given with their profs. A numerical simulation is given to test the accuracy and the applicability of our presented algorithm.
\end{abstract}

\section{Hosted file}

SubmittedPaper2020.pdf available at https://authorea.com/users/302458/articles/432530numerical-and-theoretical-treatment-based-on-the-compact-finite-difference-and-spectralcollocation-algorithms-of-the-space-fractional-order-fisher-s-equation

(a)

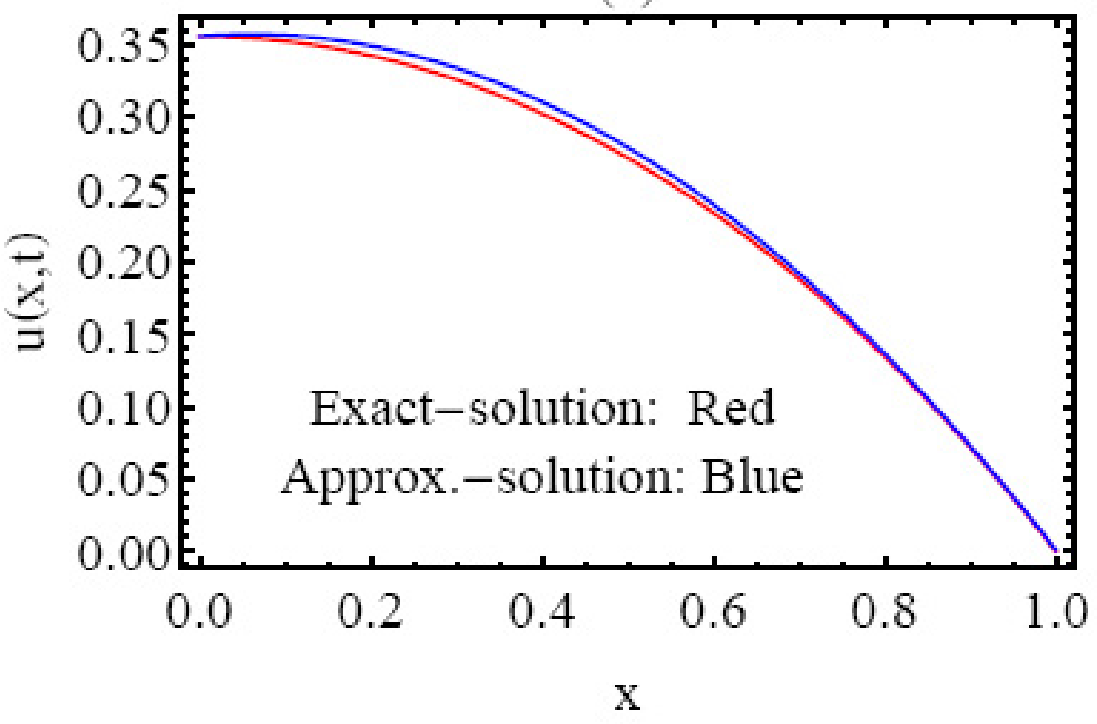


(b)

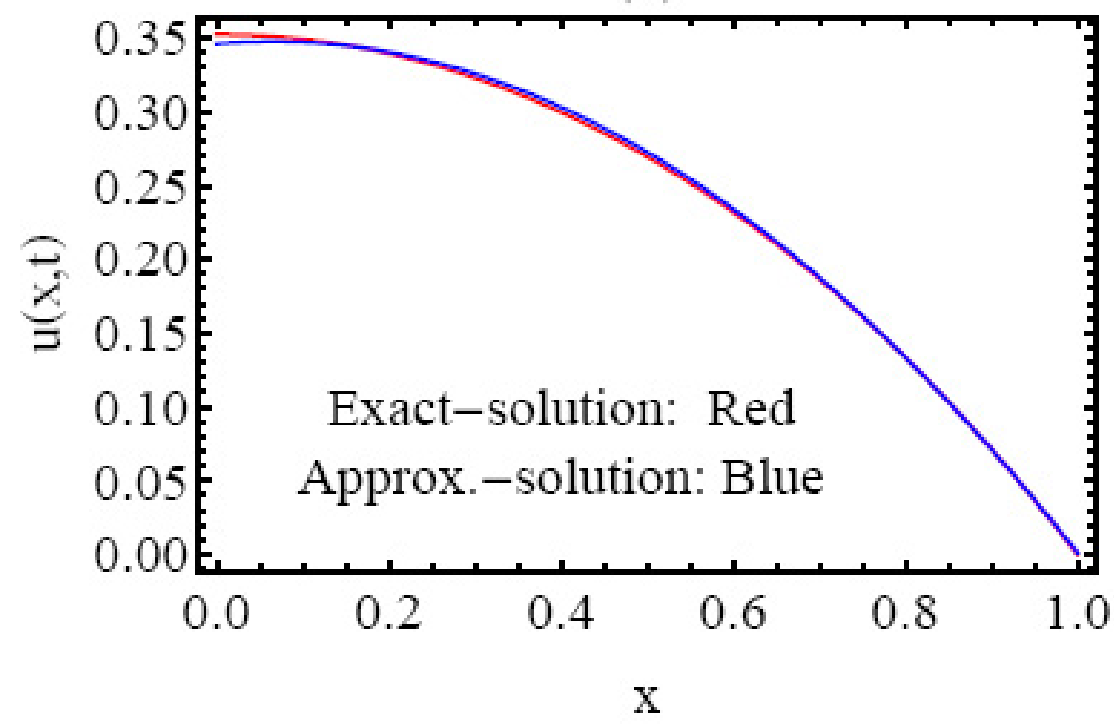

(a)

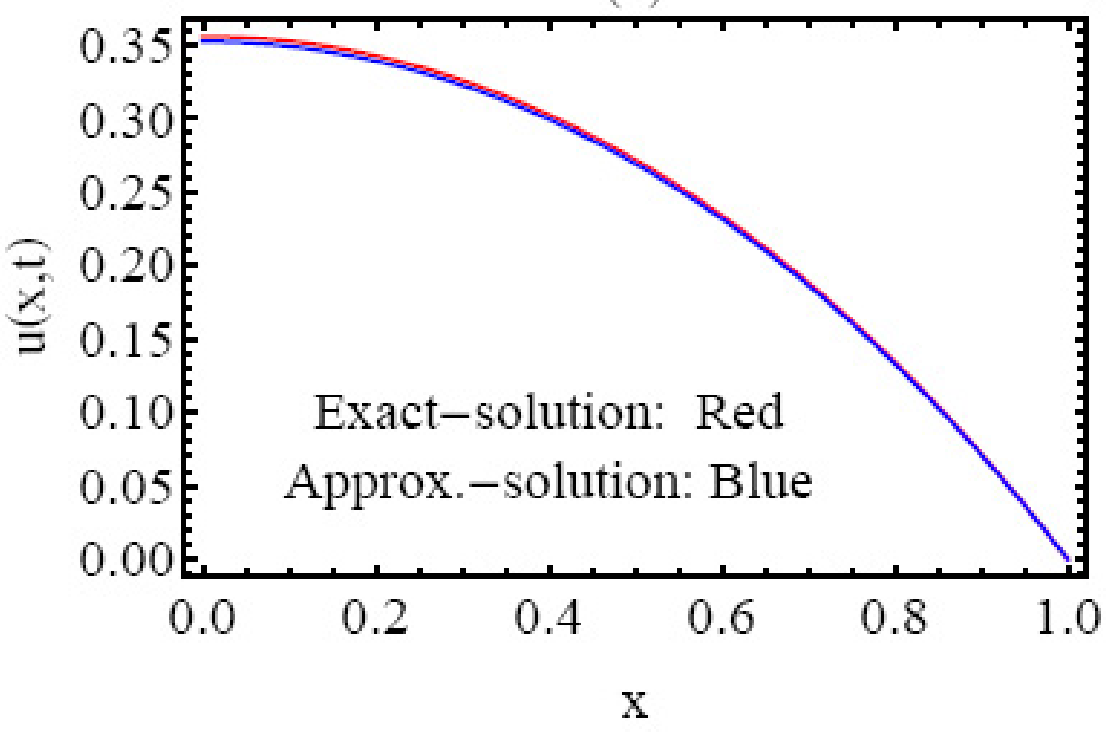


(b)

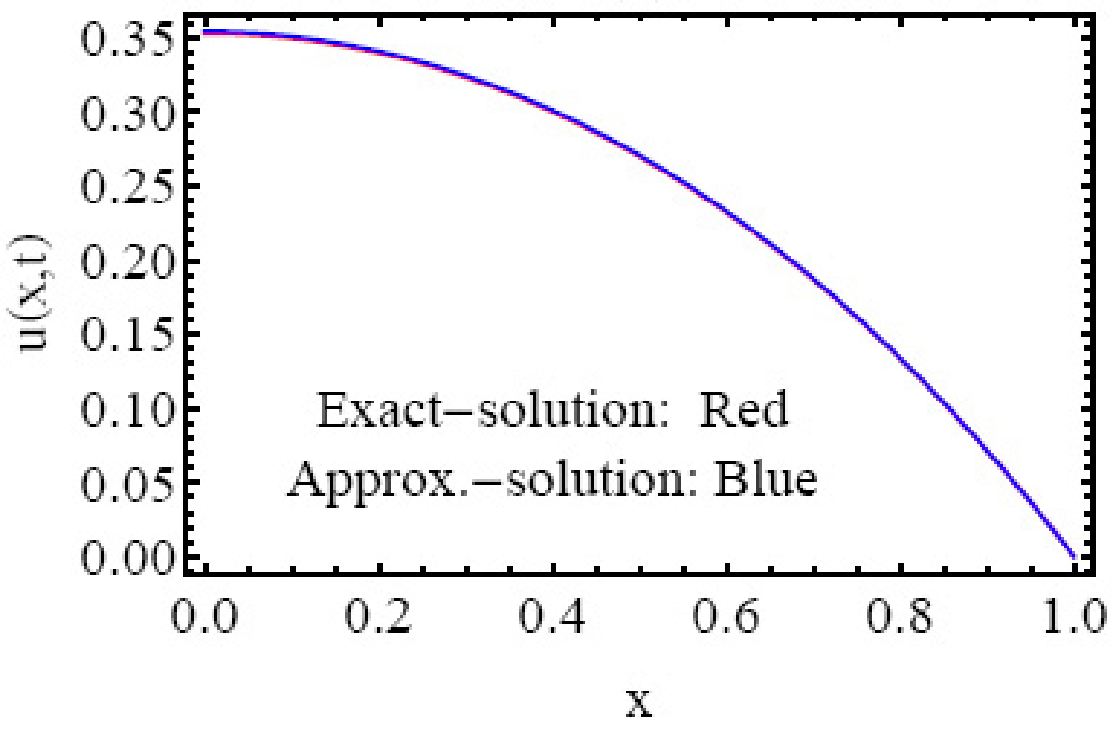

(a)

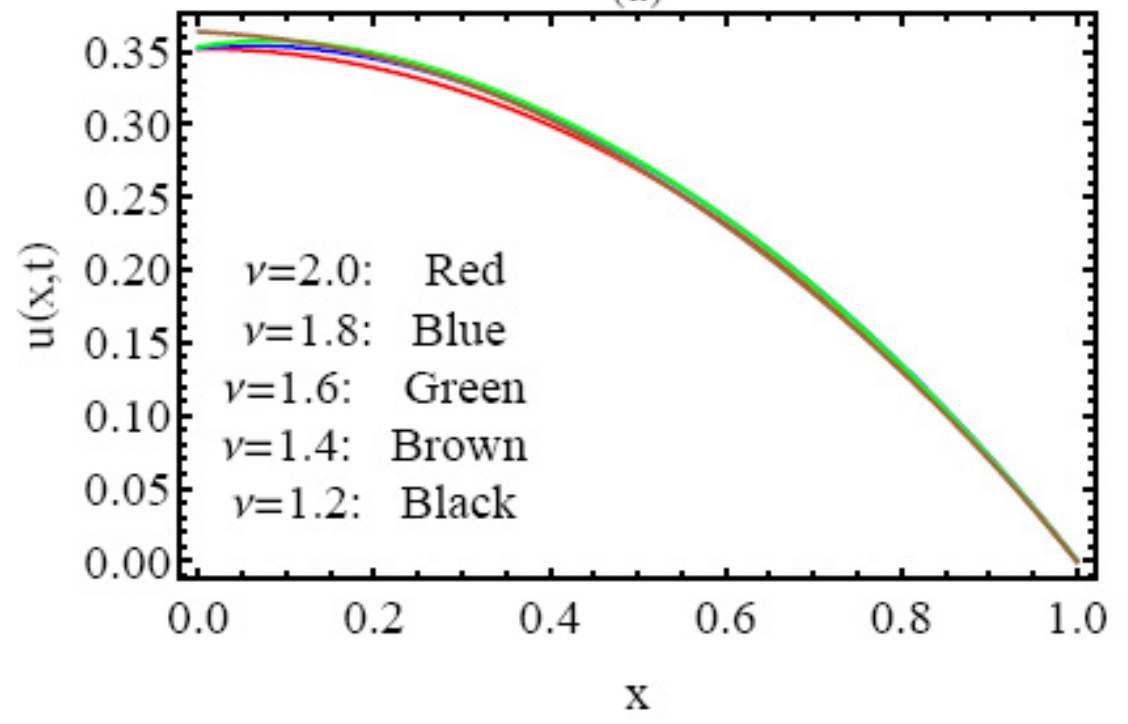


(b)

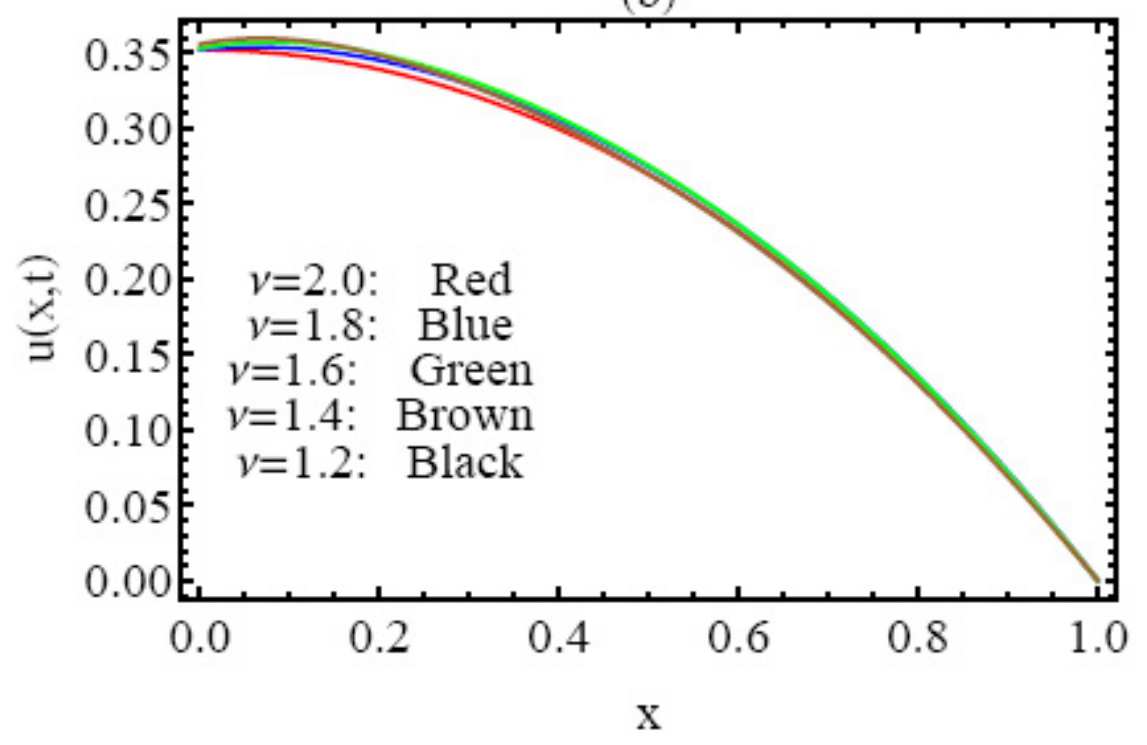

(a)

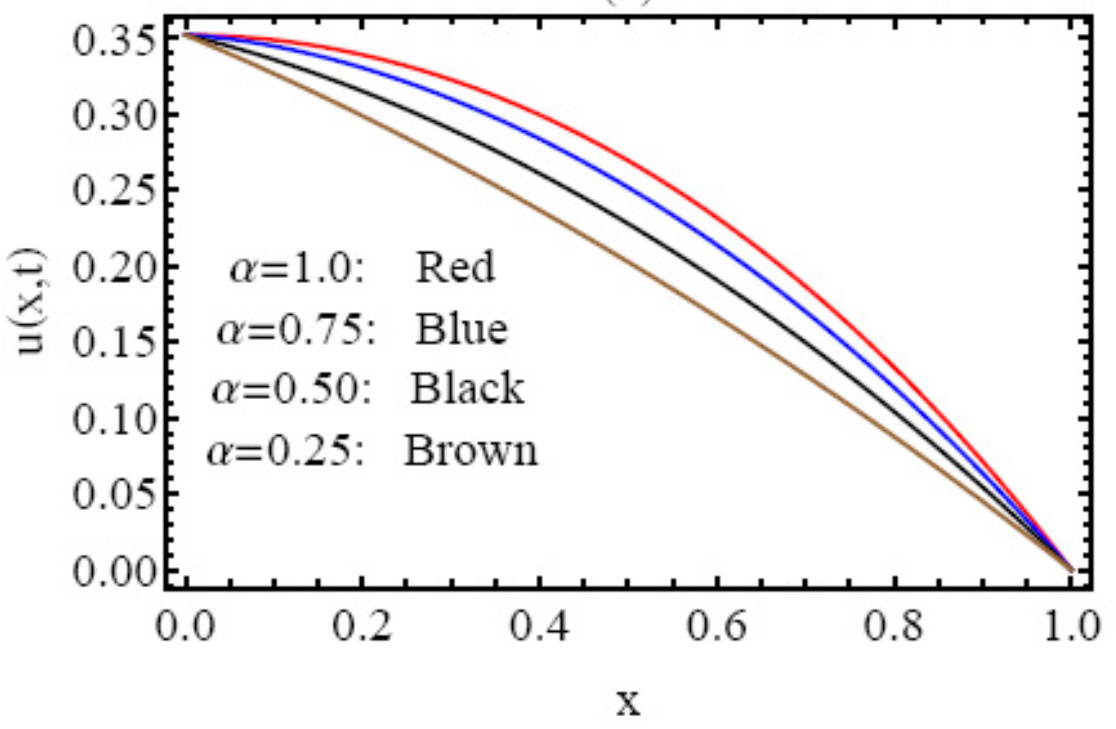


(b)

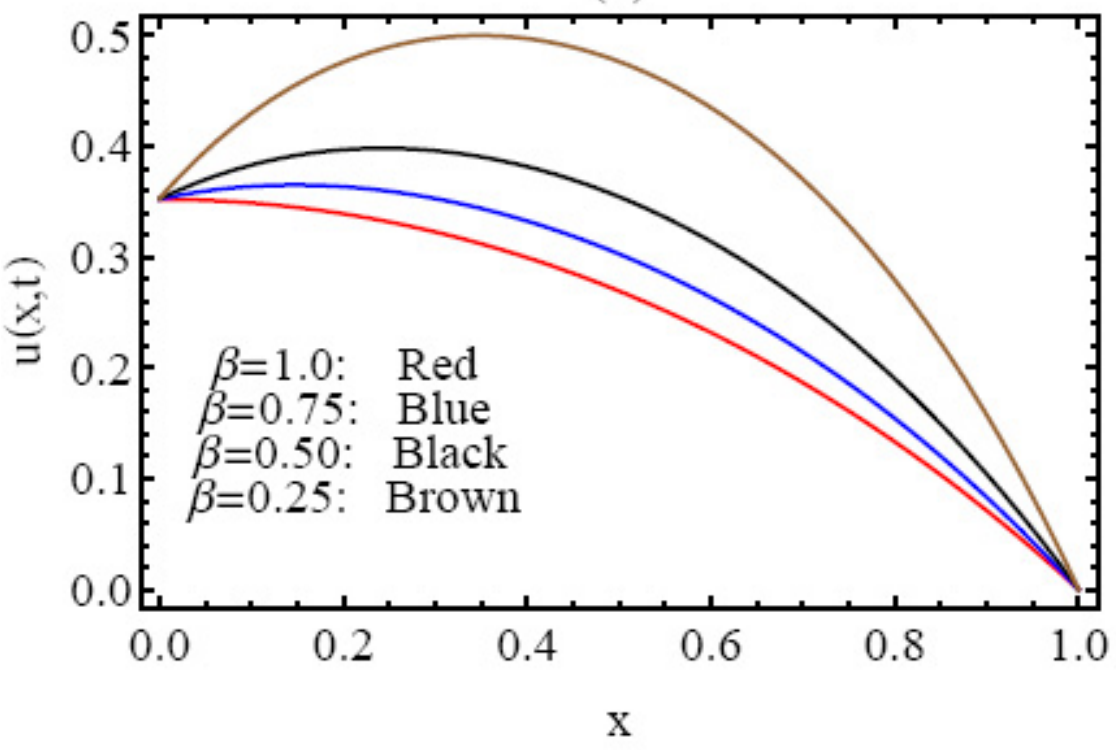

